\title{
Nutrition Metabolism
}

Adam, O., Munich, Germany

Antal, M., Budapest, Hungary

Arthington, J., Ona, Fla., USA

Berg, A., Freiburg, Germany

Bitsch, I., Giessen, Germany

Boeing, H., Bergholz-Rehbrücke, Germany

Böhm, V., Jena, Germany

Classen, H., Hohenheim, Germany

Clinton, S., Columbus, Ohio, USA

Cunningham, C., Winston-Salem, N.C., USA

De Blas, J.C., Madrid, Spain

Dobos, D., Essen, Germany

Fürst, P., Stuttgart, Germany

Gadek-Wesierski, J., Vienna, Austria

Genser, D., Vienna, Austria

Götz, M., Vienna, Austria

Grassmann, J., Munich, Germany

Hagfors, L., Umea, Sweden

Hauner, H., Munich, Germany

Hautvast, J., Wageningen, The Netherlands

Herrera, E., Madrid, Spain

Joost, H.J., Bergholz-Rehbrücke, Germany

Kafatos, A., Heraklion, Greece

Kalapos, M., Budapest, Hungary

Kar, A., Deoghar, India

Kato, Saiama, Japan

Keller, U., Basel, Switzerland

Knasmüller, S., Vienna, Austria

Koletzko, B., Munich, Germany

König, J., Vienna, Austria

Krawinkel, M., Giessen, Germany

Kudlácková, M., Bratislava, Slovak Republik

Laplace, J.P., Paris, France

Lemmens, R., Vienna, Austria

Linseisen, J., Munich, Germany

Magee, P., Belfast, UK

Marangoni, F., Milan, Italy
The editors extend their gratitude and appreciation to the following reviewers whose comments and criticisms ensure the quality of the papers published in this journal:
Martinez, A., Pamplona, Spain

Mokuda, O., Ichihara-City, Japan

Moser, U., Basel, Switzerland

Neuhäuser-Berthold, M., Giessen, Germany

Pallauf, J., Giessen, Germany

Palou, A., Palma de Mallorca, Spain

Pedersen, J., Oslo, Norway

Pool-Zobel, B., Jena, Germany

Porrini, M., Milan, Italy

Rethy, L., Budapest, Hungary

Rimbach, G.H., Reading, UK

Rodriguez-Amaya, D.B., Campinos, Brazil

Rust, P., Vienna, Austria

Salem, N., Rockville, Md., USA

Schümann, K., Munich, Germany

Simopoulos, A.P., Washington, D.C., USA

Singer, P., Bensheim-Auerbach, Germany

Sirtori, C.R., Milan, Italy

Sjöström, M., Huddinge, Sweden

Somoza, V., Garching, Germany

Stahl, W., Düsseldorf, Germany

Trichopoulo, A., Athens, Greece

Trichopoulos, D., Boston, Mass., USA

Trippo, U., Bergholz-Rehbrücke, Germany

Uauy, R., Santiago, Chile

Urbanek, R., Vienna, Austria

Villalpando, S., Cuernavaca, Mexico

Von Rücker, A., Bonn, Germany

Von Schacky, C., Munich, Germany

Wahlqvist, M.L., Melbourne, Australia

Wahrendorf, J., Heidelberg, Germany

Watzl, B., Karlsruhe, Germany

Wenk, C., Zürich, Switzerland

Winisch, W., Vienna, Austria

Wisker, E., Kiel, Germany

Zunft, H.-J., Bergholz-Rehbrücke, Germany

\section{KARGER}

(c) 2004 S. Karger AG, Basel

Fax +4161306 1234

E-Mail karger@karger.ch

www.karger.com 